\title{
PEDAGOGÍAS Y DIDÁCTICAS ESPERANZADORAS DESDE LA INTERCULTURALIDAD EN UNA UNIVERSIDAD PEDAGÓGICA NACIONAL
}

\section{Hopeful pedagogies and didactics from interculturality in a National Pedagogical University}

\author{
Saúl Alejandro-García *, \\ Felisa Yaerim López-Botello** y \\ Erika González de Salceda-Ramírez***
}

Recibido:27 de septiembre 2020. Aceptado: 01 de diciembre de 2020. Publicado: 01 de enero 2021.

Forma de citar este artículo en APA:

Alejandro-García, S., López-Botello F. Y., González de Salceda-Ramírez, E. (2021, enero-junio). Pedagogías y didácticas esperanzadoras desde la interculturalidad en una Universidad Pedagógica Nacional. Revista CoPaLa, Construyendo Paz. Latinoamericana 11 (año 6), pp. 112-125. DOI:10.35600.25008870.2021.11.0194, Recuperado desde: http://revistacopala.net/index.php/ojs/article/view/74

\section{Resumen}

Desde su creación, la Universidad Pedagógica Nacional ha tenido, la misión de regular, fortalecer y formar profesionistas en el campo de la educación. Sin embargo, el sistema educativo mexicano, enfrentan nuevos dilemas que vienen planteándose desde hace unos años atrás. En esta circunstancia, la pandemia del Covid 19 ha venido a desnudar y hacer más visible, estos temas sociales que trastocan y vulneran el sistema educativo: la violencia estructural, las desigualdades sociales, la discriminación,

\section{* Saúl Alejandro García}

Doctor en Ciencias Sociales, Maestro en Estudios Regionales y antropólogo social, miembro del SNI desde el 2015. Docente y profesor investigador de la UPN 151, Toluca, con líneas de investigación en pueblos originarios, interculturalidad, educación para la sustentabilidad y activista ambiental. Actualmente responsable de los programas y seminarios de Educación para la sustentabilidad en la UPN 151.

Orcid 0000-0002-5707-3601

otopame@yahoo.com.mx

\section{** Felisa Yaerim López Botello}

Doctora en Educación con experiencia docente desde hace 17 años, colaboro activamente en programas de Bachillerato, Licenciatura y Posgrado de la Universidad Autónoma del Estado de México. También en la Universidad Pedagógica Nacional 151, Toluca en programas de Licenciatura y Posgrado.Participación como ponente en eventos de investigación nacionales e internacionales, en publicación de revistas indexadas y arbitradas, colaboración en capítulos de libros arbitrados, elaboración de materiales y actualización de Programas, miembro del Cuerpo Académico Administración Financiera, Educación y Tecnologías de la Información de la Universidad Autónoma del Estado de México y al Colectivo de Investigadores de la Universidad Pedagógica Nacional 151, Toluca. También fui integrante del Sistema Nacional de Investigadores.

Orcid 0000-0003-1732-4979

\section{*** $\quad$ Erika González de salceda Ramírez}

Doctora en Ciencias de la Educación con experiencia docente desde hace 27 años, he participado desde docente de educación básica, Licenciatura y Posgrado de la Universidad Pedagógica Nacional 151, Toluca; en la Escuela Normal Superior del Estado de México en Posgrado únicamente. Ponente en eventos de investigación nacional e internacional, colaboración en capítulos de libros arbitrados, diseñadora y actualización de programas, elaboración de materiales, miembro fundador del del Cuerpo 117-C y al Colectivo de Investigadores de la Universidad Pedagógica Nacional 151, Toluca. También soy integrante del Programa para el Desarrollo Profesional Docente, para el Tipo Superior (PRODEP). Actualmente se desempeña como directora de la Universidad Pedagógica Nacional Unidad 151 Toluca. 
los problemas de relaciones de género, los feminicidios, los temas ambientales, entre otros.

Desde nuestro campo de acción, la educación, y del sistema educativo universitario, toca la búsqueda de establecer paradigmas que transformen la forma de pensar, actuar y vivir por parte de estudiantes y profesores. En este predicamento, se han escuchado voces de nuestra Latinoamérica y de diversos movimientos que han surgido para establecer otras formas de ver la educación. El propósito de este artículo es la reflexión de la búsqueda de pedagogías más esperanzadoras sustentadas en la interculturalidad, la pedagogía crítica y y en estudios para la paz. Pretendemos visibilizar estas prácticas pedagógicas de los profesores de la Universidad Pedagógica 151 a través de sus proyectos escolares y académicos.

\section{Palabras clave}

Pedagogías alternativas, interculturalidad, epistemologías del sur, educación neoliberal, violencia estructural

\section{Abstract}

Since its creation, the National Pedagogical University has had the mission to regulate, strengthen and train professionals in the field of education. However, the Mexican educational system faces new dilemmas that have been posed for some years now. In this circumstance, the Covid 19 pandemic has made more visible these social issues that disrupt and violate the educational system: structural violence, social inequalities, discrimination, problems of gender relations, feminicide, environmental issues, among others.

From our field of action, education, and the university educational system, it is a matter of establishing paradigms that transform the way students and professors think, act and live. In this predicament, voices have been heard from our Latin America and from diverse movements that have emerged to establish other ways of seeing education. The purpose of this article is to reflect on the search for more hopeful pedagogies based on interculturality, critical pedagogy and peace studies. We intend to make visible these pedagogical practices of the teachers of the Universidad Pedagógica 151 through their school and academic projects.

\section{Keywords}

Alternative pedagogies, interculturality, southern epistemologies, neoliberal education, structural violence Alternative pedagogies, interculturality, southern epistemologies, neoliberal education, structural violence. 


\section{Introducción}

La Universidad Pedagógica Nacional nació hace 40 años y en su desarrollo académico-formativo se han establecido directrices en materia de educación y de aportes importantes en el campo de la pedagogía. Sus licenciaturas y posgrados han permitido que miles de maestros se formen o adquieran otros conocimientos para su labor docente. Aunque en menor medida, pero de suma importancia, algunos aportes teóricos y metodológicos producto del trabajo académico y reflexivo. En estos años, la Universidad Pedagógica ha trabajado con temas y problemáticas que demanda el sistema educativo; principalmente lo relacionado con la práctica docente, procesos y ambientes de aprendizaje, cuestiones curriculares, el modelo de competencias, los aspectos de planeación y administración educativa, la psicopedagogía entre otros. Los planteamientos teóricos y metodológicos de estos temas están sustentados en los paradigmas "clásicos" de las ciencias sociales, la filosofía, la psicología entre otros, que tiene su origen en el pensamiento europeo occidental y que están presentes en los proyectos escolares, las tesis de titulación, y los programas o unidades de aprendizaje.

La naturaleza de los temas referidos se desprende de los modelos educativos que han sido implementados desde la década de los setenta hasta la actualidad (Martinez,2001; Latapi, 2004; del castillo, 2012; Leos,2019), En este sentido podemos decir que las diferentes reformas educativas han buscado las transformaciones y trascendencia de México para alcanzar el desarrollo y un mayor "bienestar” de la población. Por ello, bajo conceptos como "mejora educativa", "modernidad y modernización en la educación”, "modelo de competencias", "competitividad y calidad en la educación" se han empleado como base para establecer paradigmas educativos que se trabajan desde las instituciones formadoras de profesores, los centros educativos, dependencias gubernamentales, el magisterio (gremios sindicales), organizaciones civiles de la sociedad y la iniciativa privada.

Lamentablemente y como lo señala Leos (2019, p.25) No obstante los avances de los últimos 50 años, la aspiración de una educación de calidad, equitativa e inclusiva no ha podido cristalizarse. No hemos tenido logros completos en educación básica y mucho menos ha sido objeto de una efectiva evaluación y seguimiento que permita detectar sus aciertos, avances o retrocesos. Generalmente, cada proyecto es una visión particular de los gobernantes en turno y no hay continuidad a los planes y programas de estudios que permitan que la educación se desarrolle plenamente. A esto le agregamos dos ingredientes fundamentales: el sistema político mexicano que por décadas ha sostenido un modelo económico neoliberal, y que, en este sentido, la educación se ha convertido en un engranaje clave para seguir incursionando por el sendero capitalista, y por el otro lado, 
tenemos modelos inacabados educativos que han sido objeto de crítica por los propios actores educativos, organizaciones sociales y desde la academia. Como ejemplo, la reforma del 2013 trastocó los derechos laborales del magisterio al asociarla con la evaluación del desempeño docente, lo cual concluye con movilizaciones de algunos sectores magisteriales (Ramírez, 2017).

Resumiendo todo lo anterior, podemos decir que el modelo educativo ha estado desde hace un tiempo en crisis, al no tener una direccionalidad que le permita avanzar por encima de los proyectos políticos sexenales y que las instituciones formadoras de docentes como la UPN, se ha ceñido en trabajar bajo los quiebres y/o propuestas de los planes sexenales. Pero a pesar de ello, es desde la misma academia y de la visión del profesorado universitario, así como de algunos sectores del magisterio, que se busca establecer modelos pedagógicos y educativos alternativos que sean trascendentes y que replanteen otros caminos ante lo ya referido.

En este sentido, el trabajo que aquí presentamos tiene como propósito evidenciar a través del ejercicio reflexivo, de la experiencia de los profesores investigadores, de la multidisciplinariedad y sobre todo del compromiso social, lo que algunos autores como Souza (2010), Carbonell (2015) han denominado pedagogías alternativas, para el siglo XXI, para decolonizar el pensamiento, para desarrollar el pensamiento crítico, pedagogías para la paz entre otras. Nosotros hemos denominado pedagogías esperanzadoras.

La urgencia de estas pedagogías no es nueva, desde el año 2000 se han venido gestando críticas a los modelos económicos neoliberales y sus impactos en las sociedades humanas, esto porque hay una resistencia de las elites ricas internacionales y la política con grandes empresarios que han polarizado al mundo entre quienes tienen más y los que menos. Generando así, una crisis no solo económica, sino social, ambiental y por supuesto educativo. La pandemia del Covid 19, ha venido a visibilizar aún más la violencia estructural, las desigualdades sociales, la discriminación, los problemas de relaciones de género, los feminicidios, los temas ambientales, entre otros.

El trabajo está dividido en dos apartados: primeramente, la manera en cómo han surgido estas pedagogías sustentadas, principalmente en la interculturalidad y la paz integral. La segunda parte, mostramos algunas acciones de los docentes de la UPN 151 de Toluca por la búsqueda de otras pedagogías.

\section{1.- Pedagogías coloniales vs otras posibilidades (pedagogías esperanzadoras)}

Abramos hilo, consideramos las pedagogías coloniales cómo aquellas que están orientadas a fomentar y alimentar los modelos económicos neoliberales, a través de la educación. Quizás los lectores estén de acuerdo 
o no, acerca de la necesidad y la urgencia de mejorar los sistemas educativos nacionales, dado que, desde las perspectivas desarrollistas y de la visión de los gobiernos, la educación ha estado en crisis. El reflejo de esta situación se mira a través de indicadores de "calidad educativa" cuyos desprendimientos vienen de los organismos internacionales como la OCDE, el FMI, el Banco Interamericano de Desarrollo entre otros.

Un ejemplo de ello, han sido las evaluaciones hacia el estudiantado que se ha generado en los diversos periodos gubernamentales (desde el período de Salinas hasta la actualidad). Los ejemplos más claros han sido las evaluaciones de ENLACE, PISA, EXCALE y PLANEA que se han dado en el marco de lo que se conoce la Reforma Integral de la Educación (Leos,2019).

En este sentido, la educación en México se ha centrado en la formación básica (por recomendación de los organismos internacionales) donde se busca fomentar estrategias de aprendizaje para los estudiantes y garantizar la eficiencia terminal de estos subsistemas. La conclusión de la formación básica garantiza cierto adiestramiento y capacidades para que los jóvenes que no deseen continuar en sus estudios puedan ingresar a los sectores laborales, principalmente en los comerciales e industriales. Y los que puedan continuar, garantizar los estudios hasta el nivel superior.

En otras palabras, se busca garantizar las capacidades individuales para ser incorporados al mercado laboral (no importa el nivel de estudios) y para ello es importante tener una base educativa bien consolidada. Por esta razón, recae en los profesores del sistema básico, la responsabilidad de aplicar métodos pedagógicos que garanticen elementos mínimos de aprendizajes de los estudiantes. Principalmente en dos aspectos, en la lectoescritura para la comprensión de textos, y en el razonamiento matemático. De aquí, que el sistema educativo centre su atención en la aplicación de métodos pedagógicos que desarrollen estas habilidades en el alumnado. Y por supuesto, una gran responsabilidad de las instituciones formadoras de profesores como son las escuelas Normales y la Universidad Pedagógica Nacional.

Esto explica, los grandes debates que han generado los diferentes gobiernos y actores políticos desde la década de los ochenta a la actualidad (Latapi,2004, Del Castillo, 2012), donde las discusiones giran en torno al fracaso continuo de los modelos educativos, lo que conllevó a establecer las reformas integrales en la educación, orientadas principalmente en la educación básica.

Jurgo Torres (2019) y Dussel (2017) coinciden en señalar que hay una clara definición de los modelos pedagógicos que se llevan a cabo en las aulas. Antes del neoliberalismo las practicas educativas estaban orientadas a la formación de ciudadanos con valores y responsabilidad ética para lograr personas que pudieran 
comprometerse con la sociedad. Y los maestros de esas épocas tenían una clara convicción de ello. El neoliberalismo ha venido suplir la ética y la formación de valores, por estudiantes que desarrollen habilidades para competir en un sistema de mercado, o al menos para ser programados como consumidores. Luego entonces transformar el sistema educativo en un mercado (Torres,2019).

También señalan, que los docentes están más preocupados por que la niñez y la juventud escolar, salgan bien evaluados en las áreas correspondientes, gastando toda su energía en ello. Por ello el aprendizaje se vuelve más mecánico, más acentuado en los aprendizajes significativos; los profesores en este contexto se vuelven en transmisores del conocimiento: "bajo el propósito de enseñar conocimientos y normas, el maestro cumple la función de transmisor. El maestro dicta la lección a un estudiante que recibirá las informaciones y las normas transmitidas... El aprendizaje es también un acto de autoridad (De Zubiria, 1994, p. 15, cit. por Pinto y Castro, 2013).

En este sentido, Jurgo Torres (2019) menciona que es muy lamentable que los maestros no se den cuenta de cómo el sistema los absorbe, al punto de no tener conciencia de estas imposiciones, por lo que se vuelven presas y se convierten en piezas que sostienen al sistema. Al grado que muchos de los cambios que se realizan en las reformas educativas, los docentes ni siquiera conocen los términos o conceptos con los que se opera el modelo. Prueba de ello es que al maestro se le llama ahora "facilitador" sin tener claro lo que implica. Y lo mismo sucede cuando se habla de términos como "calidad", "competitividad", "innovación" entre otros que provienen del mundo empresarial.

Hasta aquí, queda claro que estos procederes son aplicados en las escuelas donde los docentes trabajan con los estudiantes, pero ¿qué pasa en las instituciones formadoras como las normales las Universidades Pedagógicas?

Como se comentó párrafos anteriores, las escuelas donde se forman a los profesores ha tenido un papel importante para el magisterio de este país. No tan sólo han sido responsables en la formación, sino también en la capacitación permanente de los profesores en servicio a través de la oferta de cursos, o diplomados que se fueron convirtiendo en cursos de regulación que ayudan a su formación y a su vez reflejado en la mejora del salario.

Pero cuando hablamos de la formación del estudiantado y de los currículos escolares universitarios, queda en evidencia que ha habido pocos avances en materia de actualización, investigación, innovación, mejoras en la infraestructura, en los salarios, en los sistemas de contratación y de la búsqueda de modelos pedagógicos que 
se adecuen a nuestra realidad mexicana y latinoamericana. Esto habla del poco interés por parte del estado, siendo que las instituciones formadoras, son quienes pudieran empujar por modelos y alternativas más propios de educación y depender menos de modelos internacionales ajenas a nuestra realidad.

Las reformas educativas que se han implementado en los últimos años han hecho que poco a poco haya una transición en los modelos universitarios a las instituciones formadoras de profesores. Esto es que las normales y la Universidad Pedagógica trabajen bajo los esquemas en las que opera las universidades. Un ejemplo de ello ha sido los enfoques de competencias y de los modelos de evaluación hacia los profesores, y hacia los procesos. Además de incorporar esquemas del trabajo de academias, la creación de cuerpos académicos, el programa de desarrollo al profesorado (PRODEP) que reconoce el trabajo académico y docente. En el caso de las universidades públicas, estos esquemas se han venido trabajando desde el año 2000 a través del proyecto Tunin para América Latina donde la estrategia fue impulsar el modelo de competencias genéricas para garantizar la eficacia terminal (la titulación) y dotar de herramientas que le permita al egresado competir en el mercado laboral.

Solo como comentario, este famoso modelo de competencias empezó a permear en todo el sistema educativo: empezando por las universidades y poco a poco llegar a la formación básica. Por esta razón, los conceptos relacionados con competencia, innovación, calidad se han hecho presente en las reformas educativas (Torres, 2019).

Comentábamos que, en el caso de la Universidad Pedagógica, estos esquemas empiezan a transformar los programas curriculares de las licenciaturas, pero también de los posgrados. La prueba de ello es la Maestría en educación Básica donde se trabaja de manera muy particular el enfoque de competencias para los docentes en servicio de la formación básica, pero también en las licenciaturas que se ofertan ${ }^{1}$.

Es justamente en este punto donde nos gustaría centrar el debate. Desde nuestra percepción, existe una discusión que se ha generado al interior de las universidades pedagógicas desde hace muchos años y que se refleja en la cotidianidad escolar. ¿qué tipo de profesionista es el que debemos de formar?; un estudiante que repliquen esquemas colonizadoras sustentadas en teorías funcionalistas utilizando estrategias de la psicología social como "los socioemocional", o la innovación tecnológica como medio para eficientizar procesos de aprendizaje? también el trabajo dirigido en desarrollar esquemas sustentados en la evaluación como fin último de la práctica docente?, o cómo dice Enrique Dussel (2017), que en las escuelas formadoras de profesores, se replican algunas 
estrategias para mejorar estos desempeños, pues de lo que se trata es mejorar el sistema, no hacer una crítica del sistema.

Quienes estamos inmersos en la formación de estudiantes, hemos observado que hay posturas desde las autoridades administrativas y docentes por mantener esquemas sustentadas en la calidad, la innovación, la eficiencia entre otros. En este sentido las palabras de Dussel son lógicas. Pero afortunadamente, también existen administrativos y docentes que tratan de promover modelos pedagógicos que propicien la critica a los sistemas establecidos.

Desde los años de los sesenta y setenta (incluso antes de que naciera la Universidad Pedagógica Nacional) existían movimientos sociales y políticos asociados al Marxismo, en este contexto, surge uno de los más grandes revolucionarios ideológicos que en educación vino a cimentar las bases de una pedagogía más latinoamericana, que lamentablemente su pensamiento solo ha sido retorica en muchas escuelas formadoras de docentes. Estamos hablando de Paulo Freire.

Resumiendo, a grandes rasgos la obra de Fraire (1921-1997) se centró en el humanismo y en desarrollar una pedagogía liberadora, emancipadora y a su vez esperanzadora que busca posicionar a los que menos tienen (a los no letrados y desarrapados). La posibilidad de crear una educación que permita que los individuos adquieran conciencia acerca de su realidad y de la condición humana para lograr un análisis crítico y reflexivo. Este es el camino que el educador brasileño emprendió a través de su educación popular. Él estuvo en contra de la educación bancaria que reproduce esquemas de memorización y que posiciona al maestro como una autoridad del poder. Por lo que le educación debe ser activa, e incite a ser transformadora de la realidad, sobre todo para superar la relación del opresor y del oprimido. El pensamiento de Paulo Freire contagió a una juventud que a finales de la década de los setenta y ochenta luchaban de manera abierta con los gobiernos opresores, los sistemas autoritarios y sobre todo la ideología imperialista estadounidense, que por esos años mantenía una guerra fría con la ex unión soviética.

Han pasado más de 23 años de su partida y el pensamiento de Freire sigue vigente porque:

es (...) la búsqueda de la coherencia quizá sea el principal valor, la principal virtud del Paulo que conocimos por aquellos lejanos años sesenta y setenta y que se sigue manifestando plenamente en la Pedagogía de la esperanza. Ésa es la razón por la que, viviendo con plena conciencia los tiempos actuales, no "repite" un discurso gastado, sino que rescata y reafirma sus convicciones y definiciones 
sustantivas de entonces, justamente haciéndolas valer en los tiempos actuales de tantas confusiones, de tantas derrotas y hasta claudicaciones (Nuñez,2011, p. 13).

Esta es la razón por la que Freire hereda las bases de lo que en los últimos años se ha trabajado desde la pedagogía critica con autores como Henry Hieraux, Michel Apple y Peter Maclaren, quienes actualmente empujan por una educación que forme ciudadanos críticos, pensantes, responsables y comprometidos con el género humano, y recientemente por el medio ambiente.

Si bien es cierto a Paulo Freire le toco trabajar los inicios del modelo neoliberal y cómo éste iba permeando en los sistemas económicos y educativos en Latinoamérica, ya no fue testigo de la manera en que los modelos pedagógicos neoliberales han hecho estragos en los nuevos docentes, las juventudes y niñez.

En este sentido a tocado a los autores anteriores en criticar los sistemas educativos actuales y poner énfasis que en los sistemas escolares se han convertido en instrumento de control social, ello está muy alejado de una verdadera educación, que implica el concebir al estudiante como un sujeto activo comprometido con su desarrollo y con el de la sociedad. La educación así entendida, tiene un potencial liberador que vincula a la escuela con una visión transformadora de la realidad (McLaren ,2020).

Pues bien, estos son los postulados que algunos docentes dentro de las escuelas normales y Universidades Pedagógicas trabajan. El problema es que existe una lejanía entre la teoría y la práctica, en el sentido de que lo que se discute desde estas posturas, no permea en el estudiantado y menos en los modelos de educación. Un ejemplo de ello sería tratar de trabajar una pedagogía critica bajo enfoques de competencia. Quizás habrá quienes opinen lo contrario.

Pero también hay otros caminos que posibiliten la crítica y con ello replantear la práctica docente y, sobre todo, trabajar en los contextos recientes que se han manifestado a partir de la pandemia del Covid 19 como son los temas ambientales, la salud, la alimentación, las desigualdades sociales, los valores culturales comunitarios, la marginación y otros temas que vulneran las condiciones humanas. Hacemos la aclaración que tampoco se debe olvidar el principal objetivo de la educación que tiene que ver con los temas de los aprendizajes significativos. Solo que estos deben estar orientados hacía los temas ya referidos.

Por esta razón y en los principios de Paulo Freire, tratamos de indagar en la construcción de otras posibilidades pedagógicas que de alguna manera comparten principios ontológicos y epistémicos que empujan por las 
libertades del pensamiento, recuperar lo propio (las identidades), establecer nuevas relaciones de género, la convivencia sana y pacífica, de tener una mejor relación con la naturaleza y construir una mejor vida para todos. Uno de estos conceptos que permea a lo que hemos denominado pedagogías esperanzadoras es la interculturalidad.

Las pedagogías esperanzadoras son aquellas que ubican al docente como un constructor de posibilidades, un esperanzador, comprometido con sus estudiantes donde establece procesos de aprendizaje a través del dialogo y logra mediar sus conocimientos con las de sus estudiantes, pero además invita con su ejemplo, a la reflexión a partir de los principios éticos de la vida, del derecho, del respeto a la diversidad, a la cultura, hacia la naturaleza y sobre todo del amor a sus raíces.

En este sentido y citando a Carbonell (2015) establece las pedagogías que el profesor debería tomar en consideración, estas son: pedagogía critica, pedagogías libres no directas, pedagogías de la inclusión y la cooperación, la pedagogía lenta, serena y sostenible, la pedagogía sistemática, las pedagogías del conocimiento integrado, las pedagogías de las inteligencias.

A estas habría que agregar las pedagogías interculturales, descolonizadoras, para la paz integral, pedagogías para la tierra, y todas aquellas que conlleven a otras posibilidades para tener un mundo mejor. Para algunos, todo lo mencionado no sería más que una utopía y retorica. Pero habemos quienes no creemos esto, en la Universidad Pedagógica Nacional 151 del Estado de México, un grupo de docentes y facilitados por la autoridad, han establecido estrategias que permitan al menos, establecer otras posibilidades pedagógicas que permeen en el estudiantado y por qué no, en los profesores en servicio.

\section{2.- Pedagogías esperanzadoras en la UPN 151}

Hemos llegado hasta aquí para mostrar algunas preocupaciones de como el Covid 19 ha mostrado debilidades del sistema educativo y de la necesidad de establecer propuestas emergentes que no tan sólo trunquen la formación del estudiantado, sino, además, se ha visto como una oportunidad para enfrentar desafíos que están ahogando a la sociedad en general. Temas como la educación a la distancia, los problemas de violencia estructural que se traduce en violencia de género, feminicidios, problemas de salud, del medio ambiente, entre otros, son aspectos que se han empezado a trabajar por iniciativas de docentes y autoridades.

Algunas de estas propuestas están sustentadas en estas pedagogías esperanzadoras mediante la interculturalidad. Pero antes de describir estas formas, debemos precisar en términos conceptuales, la forma en cómo se entiende lo intercultural. 
La interculturalidad con la que trabaja en algunos programas y seminarios dentro de la UPN, parten de algunos postulados de autores como Sandoval Forero (2018, 2016), Catherine Walsh (2009), Walter Mignolo (2010), Aníbal Quijano (2014), Dietz Gunther (2016), Souza (2020) por mencionar algunos. En nuestro parecer, estos autores y otros han construido un fuerte común denominado epistemologías del sur.

Las epistemologías del sur buscan, primero despojar de los procesos de enajenación social e ideológica que ha establecido la colonización neoliberal como forma de vida y que en el campo de la educación se ha alojado como un problema estructural. Segundo, interioriza a partir de los saberes propios culturales y formas de vida ancestrales como posibilidades y base para construir nuevos modelos que ayuden a reestablecer el orden social y natural. En este sentido el buen vivir se posiciona como un paradigma sustentable y educativo que puede ser una alternativa a los modelos de desarrollo, como bien se expresa:

..." las Epistemologías del Sur reflexionan creativamente sobre esta realidad para ofrecer un diagnóstico crítico del presente que, obviamente, tiene como su elemento constitutivo la posibilidad de reconstruir, formular y legitimar alternativas para una sociedad más justa y libre” (Sousa, 2011:14).

De esta manera el concepto de interculturalidad que se emplea como principio epistémico es aquel que considera el reconocimiento al pluriverso, lo cual permite conocer las formas de entender su historia, forma de ver el mundo y la manera en que se socializa con la comunidad, y sobre todo la manera en cómo se relaciona con la naturaleza. Esto es de suma importancia porque posibilita una resistencia cultural, una revitalización de las diferencias y de las particularidades de la región donde coexisten la diversidad (Viaña, Tapia y Walsh, 2010).

Es así que la interculturalidad se convierte en una posibilidad pedagógica al establecer que los procesos educativos no parten del salón de clases, sino que son compartidos con la comunidad. Es pues todo un principio de construcción social donde las relaciones sociales, culturales entre los diferentes grupos sociales son la clave. Por ello, autores como Sandoval (2018), Dietz (2011), Walsh, (2010) entre otros, sugieren que la educación intercultural es una pedagogía que permite construir acuerdos, sustentados en valores éticos que guían y norman las condiciones de convivencia social entre las poblaciones multidiversas con el propósito de establecer y de impulsar la comunalidad.

El principio de este proceso es la dialógica, cuyo planteamiento establece el reconocimiento del "otro" y de todo lo que implica como persona, como parte de una comunidad o de una mirada del cosmos diferente a la de nosotros. Esta dialógica establece un dialogo de saberes que posibilite la comunicación para reconfigurar las relaciones y los sentipensares: 
Cuando hablo de mejoramiento a través del diálogo intercultural, quiero entonces significar con ello que nosotros vamos descubriendo que nuestras propias tradiciones no bastan para ser lo que realmente quisiéramos o debiéramos ser. Tenemos la obligación, por tanto, de recuperar la memoria de lo mejor de las tradiciones con las que dialogamos como la hipoteca para el presente de esas mismas tradiciones (Fornet,2007, p. 80).

Es de esta manera que a continuación se muestran algunos de los programas, talleres y seminarios que se han empezado a trabajar en la UPN 151 a partir de la pandemia del COVID y que si bien es cierto aún no sabemos el impacto que van a generar, sobre todo porque mucho de los participantes son estudiantes, pero también hay profesores en servicio de diferentes niveles educativos.

Seminarios, talleres implementados en la UPN 151 durante el confinamiento

\begin{tabular}{|l|}
\hline $\begin{array}{l}\text { Seminario de investigación introducción de la educación para la sustentabilidad ambiental } \\
\text { en el sistema educativo: perspectivas desde el marco de los ods (objetivos de desarrollo } \\
\text { sustentable o agenda 2030). }\end{array}$ \\
\hline $\begin{array}{l}\text { Seminario de investigación la prevención temprana de la violencia en la escuela, un } \\
\text { principio para la interculturalidad. }\end{array}$ \\
\hline Seminario de investigación practicas educativas del orientador en educación. \\
\hline Seminario educación y fortalecimiento en comunidad \\
\hline Seminario de investigación psico neuroeducación, s.o.s. ante los nuevos retos en el aula. \\
\hline Seminario de investigación pedagogía crítica. \\
\hline Seminario de investigación interdisciplinaria. \\
\hline Seminario de investigación educación y valores \\
\hline Seminario de investigación equidad de género \\
\hline Taller de educación para la paz integral \\
\hline Taller de educación para la sustentabilidad \\
\hline Taller de danza y música \\
\hline
\end{tabular}

Fuente: departamento de educación continua e investigación, noviembre del 2020.

Estos seminarios y talleres han surgido como una respuesta a los problemas sociales y educativos que se han generado en los espacios escolares, pero que se han atenuado durante el confinamiento.

En el caso de los estudiantes de la Universidad Pedagógica Nacional 151 y sedes regionales, la problemática no solo se centra en los procesos de aprendizaje del estudiantado, sino en la forma en cómo están viviendo la falta de conectividad, la violencia doméstica que se genera al interior de algunos hogares derivado del machismo patriarcal, de la falta de economía originado por la violencia estructural, entre otros factores. Lo seminarios y talleres abren otras posibilidades de aprendizaje y sobre todo de aplicar estas pedagogías esperanzadoras que en mucho se necesitan para establecer otros mundos posibles. 


\section{Conclusiones}

El trabajo aquí presentado no es propiamente un trabajo de investigación, sino más bien una reflexión que busca establecer desde las epistemologías del sur (con autores como Sandoval, Dietz, Souza, Walsh y Quijano, entre otros) formas de establecer otras pedagogías transformadoras que reconozca la diversidad cultural, el pluriverso, las condiciones sociales y económicas dentro del aula, y que sean capaces de integrar a la comunidad, para hacer comunalidad, y así establecer un proceso dialógico de los profesores y de sus didácticas que desarrollan. Esto permitiría establecer y formar a ciudadanos críticos de su propia realidad, de dotar herramientas para que los jóvenes cuando sean profesores puedan incidir en proyectos de vida de sus estudiantes y por lo tanto de abrirles la posibilidad de ser felices. No crear profesores que habrán desarrollado “competencias" para integrarse a un trabajo asalariado y mal pagado.

Si en verdad aspiramos a una sociedad más democrática, más justa, con mayores oportunidades de vida digna, con una mejor relación con la naturaleza y alejados de la violencia estructural y del narcotráfico, entonces, debemos cimentar en los estudiantes y maestros de educación básica, un pensamiento crítico emancipador.

\section{Referencias bibliográficas}

DE ZUBIRIA, Julián. Tratado de Pedagogía Conceptual: Los modelos pedagógicos. Santafé de Bogotá: Fundación Merani. Fondo de Publicaciones Bernardo Herrera Merino, 1994. p. 8.

Del Castillo-Alemán G. (2012) Las políticas educativas en México desde una perspectiva de política pública: gobernabilidad y gobernanza, Magis, Revista Internacional de Investigación en Educación, vol. 4, (9), Pontificia Universidad Javeriana Colombia.

Dietz, Gunther (2016) Multiculturalismo, interculturalidad y diversidad en educación. Una aproximación antropológica, FCE, México.

Dussel Enrique (23 de agosto de 2017.) Conversatorio: "Educación, neoliberalismo y filosofía de la Liberación", Grupo de Estudios de Educación y Neoliberalismo (G.E.E.N.). Departamento de Filosofa. Facultad de Filosofía y Humanidades. Universidad de Chile. Santiago de Chile. Disponible en https://www.youtube.com/watch?v=ztTVXB-ub04\&t=4304s. consultado el 13 de noviembre del 2020.

Estermann, J. (2014). Colonialidad, descolonización e interculturalidad: Apuntes desde la Filosofía Intercultural. Polis, revista Latinoamericana, Volumen 13, No 38, pp.347-368, Santiago de Chile.

Fornet, B. R. (2006). La interculturalidad a prueba. Concordia, 43, pp. 3-70. University of Michigan

García Leos, J.L. (2019). Panorama de las reformas en educación básica. Del Plan de Once Años a la Nueva Escuela Mexicana (1970-2019). En J.A. Trujillo Holguín, A.C. Ríos Castillo y J.L. García Leos (coords.), Desarrollo Profesional Docente: reflexiones de maestros en servicio en el escenario de la Nueva Escuela Mexicana, Chihuahua, México: Escuela Normal Superior Profr. José E. Medrano R. http://www.scielo.org.mx/pdf/cpue/n24/1870-5308-cpue-24-00125.pdf 
Latapí Serra P. (2004) La política educativa del Estado mexicano desde 1992, Revista Electrónica de Investigación Educativa, vol. 6 (2), Universidad Baja California, Ensenada México. Disponible en https://redie.uabc.mx/redie/article/view/108/1408

Leiva Olivencia, J. J. (2010). La educación intercultural entre el deseo y la realidad: reflexiones para la construcción de una cultura de la diversidad en la escuela inclusiva. Revista Docencia e Investigación de la Escuela Universitaria de Magisterio de Toledo, pp.149-182, España.

Martínez Rizo F. (2001) Las políticas educativas mexicanas antes y después de 2001, Revista Iberoamericana de educación, OEI (27) disponible en https://rieoei.org/historico/documentos/rie27.htm

McLaren, Peter (2020) Presentación power point del Diplomado en Pedagogía Crítica. Instituto McLaren-UAEMéx.

Mignolo, W. y Escobar, A. (Eds.). (2010). Globalization and the decolonial option. London: Routledge.

Núñez Hurtado C. (2011) prólogo de la obra "Pedagogía de la esperanza: un reencuentro con la Pedagogía del oprimido” de Freire Paulo, Siglo XXI editores, México.

Pinto Blanco Ana M. y Castro Quitora L. (2015) una mirada crítica a los modelos pedagógicos, 1er encuentro internacional de educación "transformación del sistema educativo ecuatoriano", Facultad de Filosofía / Universidad de Cuenca-departamento de educación, disponible en https://educacionucuenca.webnode.es/news/una-mirada-critica-a-los-modelos-pedagogicos-/,

Quijano, Aníbal (2014) Colonialidad del poder, eurocentrismo y América Latina en: Cuestiones y horizontes: de la dependencia histórico-estructural a la colonialidad/ decolonialidad del poder, CLACSO, Buenos Aires.

Ramírez Díaz J.A (2017), Racionalidad, comunicación y gestión política del gobierno federal en las Reformas Educativas de 1993 y 2013, Revista de Investigación Educativa 24, Instituto de Investigaciones en Educación, Universidad Veracruzana. Xalapa.

Sandoval Forero E. A. (2018) Etnografía e investigación acción intercultural para los conflictos y la paz, metodologías descolonizadoras, EAA. Ediciones, Venezuela.

Sandoval Forero, E.A. (2016) Educación para la paz integral, memoria, interculturalidad y decolonialidad, ARFO editores, Bogotá.

Souza Santos B (2010) Descolonizar el saber, reinventar el poder, Ediciones Trilse, Uruguay.

Torres jurgo (8 de febrero 2019) conferencia Educación neoliberal, IISUE, UNAM, México, disponible en https://www.youtube.com/watch?v=S61CAF34uos \& $\mathrm{t}=1082 \mathrm{~s}$

Walsh, C. (2009). Interculturalidad, estado, sociedad. Luchas (de) coloniales de nuestra época. Quito: Abya-Yala. 